\title{
STRUCTURAL IMAGING IN THE REAL WORLD
}

\author{
Jeffrey D. Johnson \\ Amoco Production Company \\ Tulsa Research Center \\ PO Box 3385 \\ Tulsa OK \\ USA
}

\section{Introduction}

Significant advances in structural imaging/inversion during the last few years have improved our ability to delineate complex structures. 2-D poststack depth migration has been available to explorationists for some time, and 3-D poststack and 2-D prestack depth migration have now become routinely available to companies with sufficient computational resources. Several alternative algorithmic approaches have been developed that successfully accomplish inverse propagation of acoustic waves through heterogeneous media. Accurate methods that can handle steep dip and large lateral velocity changes include frequency domain approaches such as FX summation and phase shift plus interpolation, and time domain approaches such as reverse time and Kirchhoff with accurately computed traveltimes. Although implementations of each method have some contrasting advantages and disadvantages, the problem of simulating acoustic wave propagation is essentially solved for many users of imaging technologies.

The difficulties many exploration companies have encountered applying modern acoustic imaging technology in the real world, at least for standard 2-D data, include (1) seismic data quality, (2) derivation of optimum velocity models,
(3) velocity anisotropy, (4) 3-D effects on 2-D profile data, and (5) integration of geological constraints. In this short treatment I will describe a few aspects of these problems, but will not discuss many solutions.

\section{Seismic Data Quality}

Inadequate seismic data quality is a transcending problem that permeates many of our theoretically meaningful approaches to seismic exploration needs, whether they are lithologic inversion through AVO, structural imaging, or futuristic techniques such as elastic inversion. Simply stated, techniques which seem to work perfectly on ideal or model data often fail completely in the real world of seismic exploration. In the structural imaging case, many areas of complex structure also have poor seismic data quality. Some causes for poor data quality include surface noise interference, multiples, transmission effects, distortion due to near-surface complexity, and inadequate spatial sampling. The relative contribution of each of these, of course, depends on the surface and subsurface geology and the imaging objectives.

Surface noise interference is a problem seen in one form or another in virtually all of our seismic data. Particularly troublesome are land areas with hard surfaces (e.g., limestone or volcanics), or 
with large topographic relief. Shot-generated noise that is in the direction of a seismic line and does not overpower the dynamic range of our detecting or recording systems can often be handled by inline geophone arrays, proper stack arrays, and velocity filtering. Where the surface noise interference is three-dimensional in nature (often typical in complex structure areas), the problem becomes more intractable and expensive to solve. In these cases, areally deployed source and receiver arrays or wide-line profiles may be required to enhance vertically traveling signal over horizontally traveling noise.

Interference from multiple reflections is a seismic data quality problem in areas with large acoustic impedance contrasts in the near-surface or the subsurface. Simple pegleg multiples within the subsurface can often be mitigated by deconvolution; simple surface multiples, by stacking or subtraction schemes. However, many geologic environments where accurate structural imaging is needed contain interbedded high velocity layers (e.g., salt, carbonates), and complex layer geometries that result in complicated multiple wave fields that defy existing multiple suppression techniques. The Gulf of Suez and the West Africa salt basin are example areas where complex multiples inhibit accurate imaging.

Lack of penetration, excessive attenuation, or inadequate source strength are often standard scapegoats for poor seismic data quality, particularly for deep data. In many cases, the real problem is that primary signals are buried in backscattered noise or multiple reflections. In other cases (e.g., salt lenses), refraction effects create blind zones in the subsurface.

The near-surface distortion problem referred to here may require simple statics adjustments or may involve correcting for large relief topography or low velocity fill zones. These near-surface structures are best treated as part of the imaging problem. The particular difficulty is in deriving near-surface velocity models from limited aperture near-surface reflections.

\section{Velocity Modeling}

The difficulty most often cited in applying depth imaging technology, given adequate data quality and sufficient computer power, is derivation of optimal velocity models to drive imaging algorithms. Imaging practitioners often state that we really have a velocity problem, not a migration problem.

Several approaches or solutions have been suggested and tried with varying degrees of success. Stacking velocity analysis, inverse ray tracing, or tomography coupled with geological common sense are often used to build at least initial models. However, most prestack depth migrations that are currently being done seem to utilize iterative migration coupled with some form of residual velocity analysis. These residual velocity analysis techniques often involve a broad spectrum of methods generally called migration focusing analysis. Iterative techniques most often use interactive workstations networked to high performance computers. The interpreter sitting at a graphics terminal will migrate data through an initial velocity model, analyze the results, adjust the model, and then migrate data through the improved velocity model. When the velocity model is in some degree of agreement with the migrated wave field, the process is then ceased.

The iterative analysis procedures are often done by layer stripping, solving the problem layer by layer from the top downward. Focusing or residual moveout analysis helps the procedure, but in no way has been a panacea for model updating. A key for successful application of this iterative migration velocity analysis approach is the ability to accomplish very 
fast iterations, since experience shows that in some cases several tens of migration are required to obtain optimum images for a given seismic line. Another key is the direct involvement of an experienced structural interpreter who can judge the geological credibility of suggested model revisions or final answers.

However, in parameterizing velocity models a more fundamental problem may exist. Our classic view of layers with constant velocity or even layers with simple $\mathrm{X}$ and $\mathrm{Z}$ gradients may, in some cases, be questionable in light of the extreme sensitivity of prestack migrated results to minor model changes. We may need to change our model of subsurface velocities to something perhaps approximating a "cloud" of velocities where properties change continuously as a function of position. Accurate imaging in some situations may require specification of not only a macromodel of layers but a micromodel as well.

\section{Velocity Anisotropy}

Subsurface velocity models required for accurate imaging are further complicated in some environments (e.g., Tertiary shales in the North Sea) by anisotropy. Intrinsic seismic velocities in some lithologies depend on direction of propagation relative to bedding planes, fracture patterns, or other factors. In some shales, for example, horizontal velocities can be up to $50 \%$ higher than vertical velocities. Various model studies have shown that failure to include anisotropy can result in poor focusing and incorrect depth positioning.

Although we lack full understanding of the relative contributions of elliptical and nonelliptical components of anisotropy, we do know how to modify algorithms to properly account for at least elliptical components. However, what we lack is knowledge of correction factors to plug into our migration operators, and even knowledge of where inclusion may be necessary. Conventional wisdom states that anisotropic correction factors cannot be derived from surface seismic alone and require special borehole data.

\section{3-D Problems}

The problem of 2-D profile migration of reflected waves traveling in a 3-D world has been recognized since the invention of migration. 2-D prestack depth imaging seems to be more sensitive to violations of two-dimensional assumptions than conventional DMO plus time migration. Not only do we have problems with reflection sideswipe, but also propagation paths can become skewed by the three-dimensionality of velocity fields. There is some body of opinion that this flaw in 2-D techniques is fatal when dealing with very complex 3-D structures such as contorted salt lenses.

The ultimate solution is full 3-D prestack depth migration. Although the theory for this process is well known and poststack 3-D depth migration is a relatively mature technology, our available computational technology is generally lacking. Fast enough supercomputers for iterative application of the 3-D process and graphic systems for 3-D velocity model analysis are at best experimental. Particularly troublesome are the massive amounts of prestack data in even medium-sized surveys that must be fed into imaging algorithms. New disk storage technology, tape handling procedures, and multiprocessor supercomputers seem to be required.

In the meantime, selection of lines that are approximately dip to 3-D structures, application to several lines rather than one for a prospect, and involvement of structural interpreters in the process are at least intermediate steps toward prestack imaging of 3-D structures. 


\section{Geological Integration}

The bottom line for prestack depth imaging in the real world is that it is an interpretive, not a black box, process. This fact requires at least the implicit inclusion of geologic constraints and information. The problems we need to address are how to bring both hard and soft geological constraints into the velocity model building process. Some examples of geologic constraints include structure map control, well data picks, velocity limits from general lithologic information, and results from geological modeling of compaction or structural time histories. Diverse components of solutions could involve software packages for graphic workstations, development of constrained optimization algorithms, and at least multidiscipline training of the interpreter/processor individuals or teams.

\section{Conclusions}

In summary, the most important components for accurate imaging in a complex real world are: (1) adequate seismic data quality, (2) fast algorithms and computers for interactive application, and (3) knowledgeable interpreters deriving geologic models on graphics workstations. Computational speed is a major success factor. The table below gives some present day run time estimates for generic 2and 3-D imaging cases using a Cray supercomputer.

\section{Depth Migration Computer 'Time Estimates Cray Super Computer}

\author{
$10 \mathrm{Km}$ Line, $25 \mathrm{~m} \mathrm{~S}$, G Interval
}

$\begin{array}{llr}\text { V(Z) } & \text { Poststack } & 15 \text { Sec } \\ \text { V(X, Z) } & \text { Poststack } & 2 \text { Min } \\ \text { V(Z) } & \text { Prestack } & 3 \text { Min } \\ \text { V(X, Z) } & \text { Prestack } & 15 \text { Min }\end{array}$

3-D

$10 \times 10 \mathrm{Km}, 400$ Lines, 400 Shots per line

$\begin{array}{llr}\text { V(Z) } & \text { Poststack } & 3 \text { Hrs } \\ \text { V(X, Y, Z) } & \text { Poststack } & 12 \text { Hrs } \\ \text { V(Z) Y, Z) } & \text { Prestack } & \text { Days? } \\ \text { V(X, Y Y } & \text { Wrack } & \text { Wks? }\end{array}$

For the 2-D case we use 400 shot records with 120 traces per record, six seconds recording at a 4 millisecond sample rate, and a maximum frequency of about 35 Hertz. The table shows poststack migration which is truly interactive, and prestack migration which is at least semi-interactive. For the $3-\mathrm{D}$ case where we use a ten by ten kilometer area, we are dealing with 160,000 bins poststack. Here we have batch processes for poststack migration that are reasonable and cost-effective. However, for the prestack case, compute time estimates are quite unreasonable, indicating that current Cray technology is inadequate for all but small 3-D volumes.

The future of structural imaging in the real world involves solving the 3-D prestack problem and perhaps building optimization techniques which use full wave form solutions to take advantage of both amplitude and traveltime measurements. The real blue sky future, which we would hope to see ten or so years hence, involves full elastic imaging where we would like to image not only velocities and structures, but also true elastic constants. This could allow the direct identification of lithology and fluid content in the subsurface. 